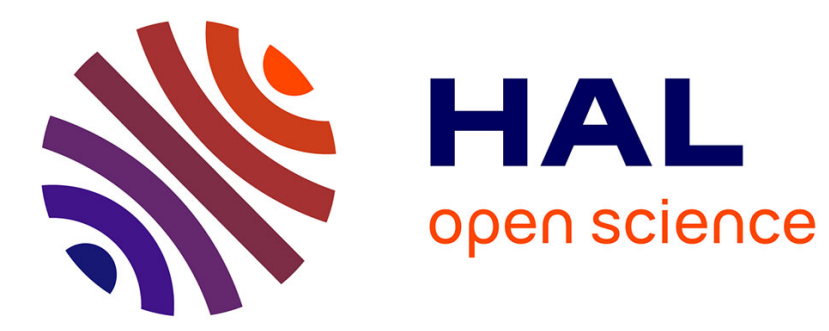

\title{
Antepenultimate broadcasting
}

Jean-Claude Bermond, Pierre Fraigniaud, Joseph Peters

\section{To cite this version:}

Jean-Claude Bermond, Pierre Fraigniaud, Joseph Peters. Antepenultimate broadcasting. Networks, 1995, 26 (3), pp.125-137. 10.1002/net.3230260302 . hal-03210206

\section{HAL Id: hal-03210206 https://hal.science/hal-03210206}

Submitted on 27 Apr 2021

HAL is a multi-disciplinary open access archive for the deposit and dissemination of scientific research documents, whether they are published or not. The documents may come from teaching and research institutions in France or abroad, or from public or private research centers.
L'archive ouverte pluridisciplinaire HAL, est destinée au dépôt et à la diffusion de documents scientifiques de niveau recherche, publiés ou non, émanant des établissements d'enseignement et de recherche français ou étrangers, des laboratoires publics ou privés. 


\title{
Antepenultimate Broadcasting*
}

\author{
Jean-Claude Bermond $†$ \\ I3S, CNRS URA 1376, Université de Nice-Sophia Antipolis, Bât. ESSI, 930 Route des Colles, B.P. \\ 145, F-06903 Sophia-Antipolis Cedex, France
}

Pierre Fraigniaud

LIP-IMAG, CNRS URA 1398, Ecole Normale Supérieure de Lyon, 46 Allée d'Italie, 69364 Lyon

Cedex 07, France

Joseph G. Peters§

School of Computing Science, Simon Fraser University, Burnaby, British Columbia, V5A 1S6, Canada

Broadcasting is an information dissemination problem in which information originating at one node of a communication network (modeled as a graph) must be transmitted to all other nodes as quickly as possible. A broadcast graph is a graph which permits broadcasting from any originator in minimum time. In this paper, we present new methods for constructing sparse broadcast graphs. Our constructions are based on graph compounding operations which are relative to vertex sets with certain properties that depend on the broadcast protocols of the graphs. We show that many previous methods for constructing sparse broadcast graphs are special cases of our methods. We demonstrate our constructions by producing new sparse broadcast graphs and by showing how many previously constructed graphs can be obtained in a systematic way.

\section{INTRODUCTION}

Broadcasting is the process of distributing information from an originator to all other nodes of a communication network. The problem addressed in this paper is that of constructing broadcast networks under the assumptions that only one piece of information is to be distributed,

* This work was done while the first two authors were visiting the School of Computing Science, Simon Fraser University. Additional support was provided by the Centre for Systems Science, Simon Fraser University.

$\dagger$ Supported by the French research program GDR-PRC C3.

$\ddagger$ Supported by the French research program GDR-PRC C3 and by the Direction des Recherches et Etudes Techniques.

$\S$ Supported by the Natural Sciences and Engineering Research Council of Canada. each communication involves exactly two adjacent nodes and takes one unit of time, and no node is involved in two or more simultaneous communications. It is easy to see that at least $\lceil\log n\rceil$ time units are required to complete a broadcast under these assumptions since the number of informed nodes can at most double during each step. The goal is to minimize the number of communication lines needed to ensure that broadcasting can be achieved in minimum time starting from any originator.

In a connected graph $G$ with $n$ vertices, a broadcast originated by a vertex $u$ determines a spanning tree rooted at $u$ called a broadcast tree or broadcast protocol for $u$. The vertices of a broadcast protocol can be labeled to indicate the times at which the vertices receive the information. The minimum time needed to complete a broadcast originated by vertex $u$ is the broadcast time of $u$ and is denoted $b(u)$. The broadcast time of $G$ is the maximum 
of the broadcast times of the vertices of $G$ and is denoted $b(G)$. If $b(G)=\lceil\log n\rceil$, then $G$ is a broadcast graph. The broadcast function $B(n)$ is the minimum number of edges in any broadcast graph with $n$ nodes, and a broadcast graph with $n$ nodes and $B(n)$ edges is called a minimum broadcast graph or $m b g$.

Minimum broadcast graphs are difficult to construct and there is no known method for constructing an $m b g$ for arbitrary $n$. Furthermore, there is no known method for determining $B(n)$ for arbitrary $n$. In fact, even determining the value of $b(u)$ for an arbitrary vertex $u$ in an arbitrary graph is NP-complete (see [16]). Since mbg's are so difficult to find, various methods for constructing sparse broadcast graphs have been proposed $[2-6,8-10,13,17-$ 19]. Most of these methods, including the new methods described in this paper, construct broadcast graphs by adding matchings or partial matchings among two or more smaller broadcast graphs. One feature that distinguishes our methods from others is the way in which we exploit properties of the broadcast protocols of graphs to reduce the sizes of the partial matchings.

The compound of a graph $G$ into a graph $H$ is obtained by replacing each vertex of $H$ by a copy of $G$ and each edge of $H$ by a matching between the vertices of the two corresponding copies of $G$. Our new construction methods use compounds relative to carefully chosen subsets of vertices of $G$. In these compounds, the matchings corresponding to the edges of $H$ are restricted to edges with endpoints in the chosen subsets of vertices.

Our starting point is a method of Khachatrian and Harutounian [10] in which the chosen subset is a vertex cover for $G$ (i.e., every edge of $G$ has at least one endpoint in the subset), $H$ is $K_{2}$, and the maximum degree in $G$ is bounded by $\lceil\log n\rceil-1$ (where $n$ is the number of vertices in $G$ ). In Section 2, we present a more general version of this construction in which we replace the maximum degree restriction on $G$ with a condition that there exist broadcast protocols in which certain vertices are idle at certain times. We then further generalize the method to allow $H$ to be any broadcast graph. Of course, a vertex cover for $G$ is a cover of all paths of length 1. In Section 3 , we show how to modify the conditions about idle vertices so that subsets which are covers of all paths of length 2 (2-path covers) can be used. Our constructions can be further generalized to $h$-path covers for any $h>2$. We use $h$-path covers to construct families of broadcast graphs on $n=2^{k}-2^{j}$ vertices in which the difference between maximum and minimum degrees can be varied between 0 and $j-1$.

Some previous methods are not special cases of our $h$ path cover methods for any $h$. Farley [6] proposed methods which construct broadcast graphs by adding matchings among two or three smaller broadcast graphs. Farley's constructions are not compounds in the sense described above because the smaller broadcast graphs can be dif- ferent. Farley's constructions were generalized by Chau and Liestman [3] to obtain the 5-way, 6-way, and 7-way split methods. In Section 4, we present the $k$-way split method which generalizes the constructions of [3], and we determine the conditions under which a $k$-way split could give better results than can our method based on 2-path covers. We have not found any cases for which these conditions are satisfied.

In Section 5, we show that many previous constructions are special cases of our new constructions. Then, we illustrate our methods by presenting an annotated table of the sparsest known broadcast graphs on $n$ vertices for $n$ $<64$ and a sample of new results for larger values of $n$. For many values of $n$ for which mbg's were not previously known, our new methods produce the sparsest-known graphs.

\section{PARTIAL MATCHINGS AND SOLID 1-COVERS}

In [10], Khachatrian and Harutounian showed that a broadcast graph with $2 n$ vertices can be constructed from a broadcast graph $G$ with $n$ vertices and maximum degree $\lceil\log n\rceil-1$ by finding a vertex cover $S$ for $G$ and then joining two copies of $G$ with a matching that joins each vertex in the vertex cover of one copy of $G$ with its image in the other copy of $G$. (Recall that a vertex cover of a graph is a subset of vertices that includes at least one endpoint of each edge.) Theorem 1 below is a generalization of their result in which we replace the maximum degree condition with a condition involving idle vertices and solid 1-covers.

Definition 1. Given a broadcast protocol in a broadcast graph $G$ on $n$ vertices, a vertex $u$ is idle at time $t \leq\lceil\log$ $n\rceil$ if and only if $u$ is aware of the message at (the beginning of) time step $t$ and $u$ does not communicate with any of its neighbors during time step $t$.

For example, in any broadcast protocol for $C_{6}$, the first informed vertex is idle at time 3 . For $C_{5}$, there is a broadcast protocol in which the first informed vertex is idle at time 2. Note that being idle at time $t$ does not imply being idle at any time greater than $t$.

Definition 2. Given a broadcast graph $G$, a subset of vertices $C$ is $a$ solid 1-cover if and only if $C$ is a vertex cover of $G$, and for each $u \notin C$, there is a broadcast protocol for $u$ such that at least one neighbor of $u$ is idle at some time during the broadcast.

For any $n>2, n \neq 4$, there is a solid 1-cover of $C_{n}$ of size $\lceil n / 2\rceil$. There is no solid 1-cover of $C_{4}$ of size less than 4. In fact, there is no solid 1-cover of size less than $2^{d}$ in 


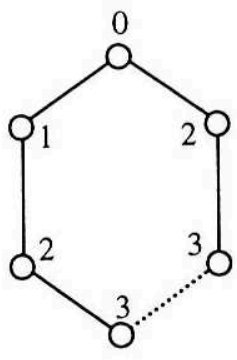

(a)

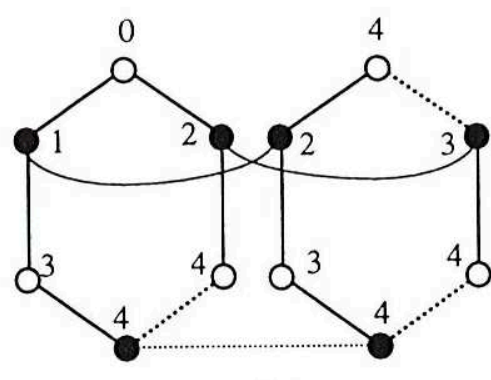

(b)
Fig. 1. A graph and broadcast protocol based on a solid 1cover.

the $d$-dimensional hypercube for any $d$ because there are no idle vertices in any broadcast protocol for a hypercube. Also, in any graph on $n$ vertices with maximum degree $\lceil\log n\rceil-1$, any vertex cover is a solid 1 -cover, and, in this special case, the following theorem and proof reduce to results in [10]:

Theorem 1. Let $G=(V, E)$ be a broadcast graph on $n$ vertices. If $C$ is a solid 1-cover of $G$, then $B(2 n) \leq 2|E|$ $+|C|$.

Proof. Let $G^{\prime}$ be the graph constructed from two isomorphic copies $G_{1}$ and $G_{2}$, of $G$, by joining these copies with a perfect matching between $C_{1}$ and $C_{2}$ (each vertex $u_{1} \in C_{1}$ is joined to its "copy" $\left.u_{2} \in C_{2}\right)$. $G$ ' has $2 n$ vertices and $2|E|+|C|$ edges. We show that $b\left(G^{\prime}\right)=\lceil\log n\rceil+1$.

Let $u_{1} \in V\left(G_{1}\right)$ be the originator of the broadcast.

If $u_{1} \in C_{1}$, then $u_{1}$ sends the message to its copy $u_{2}$, and then $u_{1}$ and $u_{2}$ broadcast in $G_{1}$ and $G_{2}$, respectively. This takes $\lceil\log n\rceil+1$ time steps.

Suppose that $u_{1} \notin C_{1}$, and let $\mathcal{P}$ be any broadcast protocol for $u_{1}$ in $G_{1}$. Let $v_{1}(i), i=1,2, \ldots$ be the neighbors of $u_{1}$ in $G_{1}$ that are informed by $u_{1}$ at times $i=1,2, \ldots$. Since $C_{1}$ is a vertex cover, all of the $v_{1}(i)$ 's belong to $C_{1}$. We can extend $\mathcal{P}$ to inform the vertices of $G_{2}$ as follows: When a $v_{1}(i)$ receives the message, it first sends it to its copy $v_{2}(i) \in G_{2}$. Then, $v_{1}(i)$ and $v_{2}(i)$ both complete broadcasts according to $\mathcal{P}$ in $G_{1}$ and $G_{2}$, respectively. Thus, all vertices of $G_{1}$ and all vertices of $G_{2}$ except $u_{2}$ will be informed after $\lceil\log n\rceil+1$ time steps. Since $C$ is a solid 1-cover of $G$. we can choose $\mathcal{P}$ to be a broadcast protocol which ensures that some neighbor of $u_{2}$ in $G_{2}$ is idle at some time step during the broadcast and this vertex can inform $u_{2}$ during its idle time step.

Since $C_{6}$ has a solid 1-cover of size 3 , the theorem shows that $B(12) \leq 15$. Figure 1 (a) shows a broadcast protocol for $C_{6}$ and Figure 1(b) show's the 12 vertex graph and broadcast protocol that result when the construction of Theorem 1 is applied. Of course, we already know that $B(12)=15$, and the graph in Figure $1(b)$ is a graph which appears in [18]. Figure 2 shows a solid 1-cover of cardinality 7 of a broadcast graph on 14 vertices. Therefore. $B(28) \leq 49$, duplicating a result in [10] and improving on the best previously known bound of $B(28) \leq 52$ [2]. There are many applications of Theorem 1 that improve previously known bounds on $B(n)$. We refer to Table I for other applications.

Theorem 1 is a special case of the following more general result based on a graph compounding operation which is relative to solid 1 -covers.

Definition 3. Let $G=(V(G), E(G))$ and $H=(V(H), E(H))$ be two graphs. The compound of $G$ into $H$ relative to a set $S \subseteq V(G)$, denoted $G_{S}[H]$, is obtained by replacing each vertex $x$ of $H$ by a graph $G_{x}$ isomorphic to $G$ and adding a matching between two sets $S_{x}$ and $S_{y}$ if $x$ and $y$ are adjacent in $H$. More precisely, the matching between $S_{x}$ and $S_{y}$ connects each vertex in $S_{x}$ with its copy in $S_{y}$. For any vertex $u \in S$, we use $H_{u}$ to denote the graph isomorphic to $H$ which interconnects the copies of $u \in V(G)$.

Theorem 2. Let $G=(V(G), E(G))$ and $H=(V(H), E(H))$ be broadcast graphs on $n$ and $k$ vertices, respectively, and let $C$ be a solid 1-cover of $G$. If $\lceil\log k n\rceil=\lceil\log k\rceil+\lceil\log$ $n 7$, then $G_{\mathrm{C}}[H]$ is a broadcast graph and, therefore,

$$
B(k n) \leq k|E(G)|+|C||E(H)| .
$$

Proof. $G_{C}[H]$ has $k n$ vertices and $k|E(G)|$ $+|C \| E(H)|$ edges. We need to show that $b\left(G_{C}[H]\right)$ $=\lceil\log k n\rceil=\lceil\log k\rceil+\lceil\log n\rceil$. Let $u_{x} \in V\left(G_{x}\right)$ be the originator of the broadcast, i.e., $u_{x}$ is the copy of $u \in V(G)$ in $G_{x}$ for some $x \in V(H)$. Let $\mathcal{P}$ be a broadcast protocol for vertex $u$ in $G$, and $Q$, a broadcast protocol for vertex $x$ in $H$.

If $u_{x} \in C_{x}$, then $u_{x}$ uses $Q$ to broadcast in $H_{u}$ during the first $\lceil\log k\rceil$ time steps. During the next $\lceil\log n\rceil$ time steps, each copy of $u$ uses $\mathcal{P}$ to broadcast in its corresponding copy of $G$ to complete the broadcast in $G_{C}[H]$ in $\lceil\log k\rceil+\lceil\log n\rceil$ time steps.

If $u_{x} \notin C_{x}$, then $u_{x}$ performs its broadcast in $G_{x}$ using a protocol $\mathcal{P}$ for $u$ in $G$ which ensures that $u$ has an idle

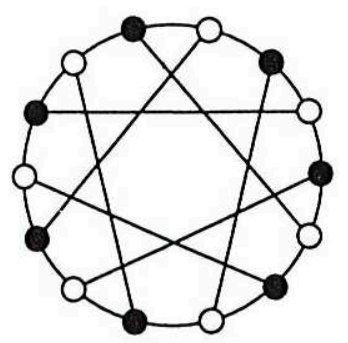

Fig. 2. A solid 1-cover of a broadcast graph on 14 vertices. 
neighbor at some time during its broadcast. It is always possible to choose such a $\mathcal{P}$ because $C$ is a solid 1 -cover of $G$. Let $v_{x}(i), i=1,2, \ldots$ be the vertices of $G_{x}$ informed by $u_{x}$ at times $i=1,2, \ldots$ Since $C_{x}$ is a vertex cover, all of the $v_{x}(i)$ 's belong to $C_{x}$. When a $v_{x}(i)$ receives the message from $u_{x}$, it first uses protocol $Q$ to broadcast in $H_{v_{x}(i)}$ and then completes its broadcast in $G_{x}$ according to $\mathcal{P}$. Each vertex of $H_{v_{x}(i)}$, upon receiving the message, first completes the broadcast in $H_{v_{x}}(i)$ according to $Q$ and then completes a broadcast in its copy of $G$ according to protocol $\mathcal{P}$. This broadcast protocol for $G_{C}[H]$ ensures that all vertices of $G_{C}[H]$, except the copies of the originator $u_{x}$, are informed after $\lceil\log k\rceil+\lceil\log n\rceil$ time steps. Furthermore, each copy of $u_{x}$ has a neighbor which is idle at some time step during the broadcast, and these neighbors can inform the copies of $u_{x}$ during their idle time steps.

Again, we can recover previously known values of $B(n)$. For example, the mbg on 3 vertices has a solid 1-cover of 2 vertices. When $G$ and $H$ are both the mbg on 3 vertices, our construction yields an mbg with 9 vertices and 10 edges which appears in [6]. More importantly. there are many applications of Theorem 2 that improve previously known bounds on $B(n)$. For example, when $G$ and $H$ are the mbg's on 6 and 7 vertices, respectively, we obtain $B(42) \leq 66$. whereas the best previously known bound was $B(42) \leq 80$ [2]. Of course, Theorem 1 is the special case of Theorem 2 with $H=K_{2}$. See Table I for other applications.

We can also use Theorem 2 to find infinite families of broadcast graphs. The infinite family of mbg's with $n=2^{k}-2$ vertices $[5,10]$ is a family of bipartite graphs of maximum degree $k-1$ and establishes that $B\left(2^{k}\right.$ $-2)=(k-1)\left(2^{k-1}-1\right)$. These graphs were used by Khachatrian and Harutounian [10] to construct broadcast graphs with $2^{k}-2^{j}$ vertices. Their result is a simple corollary of Theorem 2 .

Corollary 1 (Khachatrian and Harutounian). $B\left(2^{k}-2^{\jmath}\right)$ $\leq\left[\left(2^{k}-2^{j}\right) / 4\right](2 k-j-1)$ for an $1 \leq j \leq k-2$ and $k$ $\geq 4$.

Proof. The family of mbg's with $n=2^{k}-2$ vertices establishes the result for $j=1$. For $j>1$, let $G$ be a regular bipartite $\operatorname{mbg}$ on $n=2^{k-j+1}-2$ vertices. Either component of the bipartition of $G$ is a vertex cover. Furthermore, any vertex cover is a solid 1-cover because the degree is $\lceil\log n\rceil-1$. Applying Theorem 2 with $|C|=2^{k-j}-1$ and $H$ a hypercube of dimension $j-1$ gives the result.

The graphs on $2^{k}-2^{j}$ vertices that result from Corollary 1 are not regular since half of the vertices are of degree $k$ $-j$ and the other half are of degree $k-1$. In the next section, we use a more sophisticated construction to find new families of graphs on $2^{k}-2^{\jmath}$ vertices with smaller differences between the maximum and minimum degrees.

Theorem 2 also gives a new upper bound on $B(k n)$ in terms of $B(k)$ and $B(n)$.

Corollary 2. If $n$ and $k$ are integers such that $\lceil\log k n\rceil$ $=\lceil\log k\rceil+\lceil\log n\rceil$, and $\gamma(n)$ is the size of the smallest solid 1-cover of any mbg on $n$ vertices, then

$$
B(k n) \leq k B(n)+\gamma(n) B(k) .
$$

The upper bound of Theorem 2 is not always minimized by choosing $G$ to be an mbg. For large $k$ especially, it is more important to minimize the size of the solid 1cover of $G$ than the number of edges in $G$. For example, all mbg's on 7 vertices have 8 edges and must have solid 1 -covers of size at least 5 , since a smaller solid 1-cover would give $B(14)<21$ and we know that $B(14)=21$. Figure 3(a) shows a solid 1-cover of size 5 of an mbg on 7 vertices. Applying the formula from Corollary 2 with $n$ $=k=7$ gives $B(49) \leq 7 B(7)+5 B(7)=96$, which is better than the best previous value 99 [2]. However, Figure 3(b) shows a solid 1-cover of size 4 of a broadcast graph on 7 vertices which is not an mbg because it has 9 edges. Applying Theorem 2 with $n=k=7$ gives $B(49) \leq 7 \times 9$ $+4 B(7)=95$. See Table $I$ for other applications.

Remark. Since $G$ and $H$ do not play the same role in Theorem 2, compounding is neither a commutative operation nor an associative operation. For example, compounding a broadcast graph with 7 vertices into the $\mathrm{mbg}$ with 6 vertices requires at least 78 edges, whereas compounding in the other order uses 66 edges.

\section{SOLID 2-COVERS AND SOLID $h$-COVERS}

The conditions in the previous section based on solid 1covers ensure that all copies of the originator can be informed. Stated another way, if the originator $u$ is not in the solid 1-cover, then there is a broadcast tree (protocol)

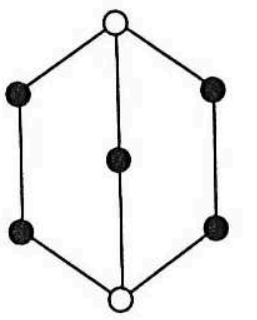

(a)

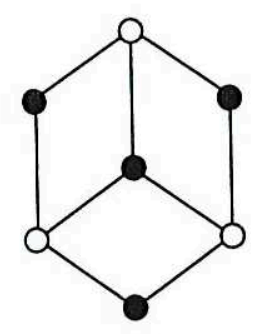

(b)
Fig. 3. Solid 1-covers of broadcast graphs on 7 vertices. 
$\mathcal{P}$ rooted at $u$ in which each copy of $u$ has at least one neighbor which has an idle time unit sometime during the broadcast. In this section, we generalize these conditions so that there are enough idle vertices in $\mathcal{P}$ to inform all copies of the originator and all copies of one of its neighbors. These generalized conditions are based on solid 2-covers. We then use solid h-covers and generalize the conditions further so that there are enough idle vertices to inform all copies of the vertices on a path through $u$ of length at most $h-1$.

Definition 4. Given a broadcast graph $G$ on $n$ vertices, a subset of vertices $M$ is a solid 2-cover if and only if $M$ covers all the paths of length 2 in $G$, and for each $u \notin M$, there is a broadcast protocol from u such that at least one of the following three conditions holds:

1. All neighbors of $u$ belong to $M$ and at least one neighbor of $u$ is idle at some time during the broadcast.

2. Exactly one neighbor $v$ of $u$ does not belong to $\mathrm{M}$, at least one of the neighbors of u distinct from $v$ is idle at some time during the broadcast. and at least one of the neighbors of $u$ distinct from $\|$ is idle at some time during the broadcast.

3. Exactly one neighbor $v$ of $u$ does not belong to .M and at least one of the neighbors of $u$ distinct from $v$ or at least one of the neighbors of $v$ distinct from $u$ is idle at some time $t<\lceil\log n\rceil$ during the broadcast.

For example, Figure 4 shows a solid 2-cover of size 2 for $C_{5}$ and a solid 2-cover of size 3 for an mbg with 9 vertices. The vertex labeled $x$ in Figure $4(a)$ has a broadcast protocol for which the solid cover satisfies condition 3 of Definition 4. The vertices labeled $y$ and $z$ in Figure 4(b) have broadcast protocols for which the solid cover satisfies conditions 2 and 1, respectively. Matching with solid 2covers is often more efficient than with solid 1-covers since solid 2-covers of graphs are often smaller than solid 1covers.

Theorem 3. Let $G=(V(G), E(G))$ and $H=(V(H), E(H))$ be broadcast graphs on $n$ and $k$ rertices, respectively: and let $M$ be a solid 2-cover of $G$. If $\lceil\log k n\rceil=\lceil\log k\rceil$ $+\lceil\log n\rceil$, then $G_{M}[H]$ is a broadcast graph and, therefore,

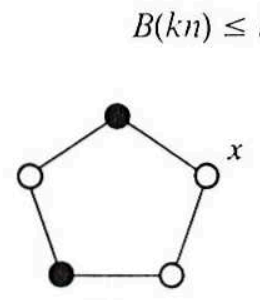

(a)

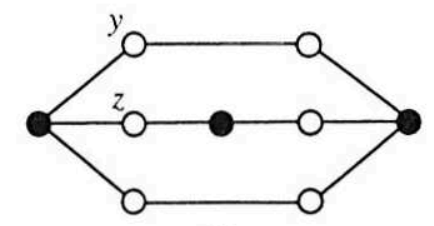

(b)
Fig. 4. Solid 2-covers of mbg's on 5 and 9 vertices.

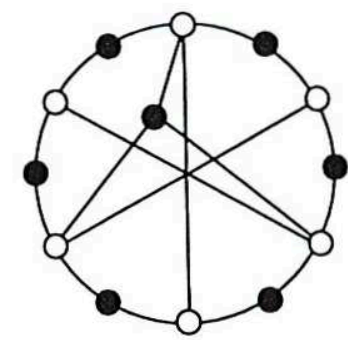

Fig. 5. Solid 2-cover of an mbg on 13 vertices.

Proof. The proof is similar to the proof of Theorem 2. $G_{M}[H]$ has $k n$ vertices and $k|E(G)|+|M||E(H)|$ edges, so we need to show that $b\left(G_{M}[H]\right)=\lceil\log k n\rceil=\lceil\log k\rceil$ $+\lceil\log n\rceil$. Let $u$ be the originator of the broadcast. If $u$ belongs to $M$, then we can use the same arguments as in the proof of Theorem 2.

Suppose that $u$ does not belong to $M$. Since $M$ is a solid 2-cover of $G$, one of the three conditions in Definition 4 holds. If the first condition is true, then all neighbors of $u$ belong to $M$ and we can use the same arguments as in the proof of Theorem 2. If the second or third condition is true, then the same broadcasting protocol as in the proof of Theorem 2 can be used to ensure that all vertices, except the copies of $u$ and the copies of one of its neighbors $v \notin M$, are aware of the message at time $\lceil\log k\rceil+\lceil\log n\rceil$. Since $M$ is a solid 2-cover, there are enough idle vertices to inform all copies of $u$ and $v$ by the end of time step $\lceil\log k\rceil+\lceil\log n\rceil$.

As with Theorems 1 and 2, we can recover previously known values of $B(n)$. For example. Theorem 3 with $H$ $=K_{2}$ can be used with the graph of Figure 4(a) to construct an mbg on 10 vertices which appears in [14]. Theorem 3 with $\mathrm{H}=\mathrm{K}_{2}$ and the graph of Figure 4(b) gives an mbg on 18 vertices which appears in [4]. With $G=C_{5}$ and $H$ the mbg on 7 vertices in Figure 3(a), we obtain a new value $B(35) \leq 51$, whereas the best previously known bound on $B(35)$ was 54 [2]. Figure 5 shows a solid 2-cover of size 7 for an mbg on 13 vertices. Therefore, $B(26) \leq 43$. whereas the best previous bound was 48 [2]. We refer to Table I for other applications.

Theorem 3 gives another new upper bound on $B(k n)$ in terms of $B(k)$ and $B(n)$.

Corollary 3. If $n$ and $k$ are integers such that $\lceil\log k n\rceil$ $=\lceil\log k\rceil+\lceil\log n\rceil$, and $\mu(n)$ is the size of the smallest solid 2-cover of any $m b g$ on $n$ vertices, then

$$
B(k n) \leq k B(n)+\mu(n) B(k)
$$

The constructions of Theorems 1 and 3 can be combined to give a family of regular broadcast graphs with $2^{k}$ 
8 vertices. The family of graphs is the same as the family found by Mahéo and Saclé [13]. However, our proof below is considerably simpler than is the proof in [13].

Corollary 4 (Mahéo and Saclé). There are regular broadcast graphs of degree $k-2$ with $2^{k}-8$ vertices and $\left[\left(2^{k}\right.\right.$ $-8) / 2$ ] $(k-2)$ edges for $k \geq 5$.

Proof. Let $G$ be a regular bipartite mbg on $n=2^{k-2}$ - 2 vertices. Recall that either component of the bipartition is a solid 1-cover because $G$ has degree $\lceil\log n\rceil-1$. Let $S$ be one of the components and apply Theorem 1 to construct a graph $G^{\prime}$ on $n^{\prime}=2^{k-1}-4$ vertices. This graph is composed of two copies, $G_{1}$ and $G_{2}$, of $G$, connected with a perfect matching between the copies, $S_{1}$ and $S_{2}$, of $S$ (see Fig. 6). We claim that the set $\overline{S_{1} \cup S_{2}}=\left(V\left(G_{1}\right)\right.$ $\left.-S_{1}\right) \cup\left(V\left(G_{2}\right)-S_{2}\right)$ of vertices not involved in the perfect matching is a solid 2-cover of $G^{\prime}$. To see this, first notice that every vertex in $S_{1} \cup S_{2}$ has all but one neighbor in $\overline{S_{1} \cup S_{2}}$. Now, since all of the vertices in $\overline{S_{1} \cup S_{2}}$ have degree $\left\lceil\log n^{\prime}\right\rceil-2$, the third condition of Definition 4 holds. The result now follows by Theorem 3 using $G=G^{\prime}$ and $H=K_{2}$.

The construction in the proof of Corollary 4 can be generalized to give new families of graphs with $2^{k}-2^{j}$ vertices by using solid $h$-covers for $h>2$. We will not give a formal definition of the conditions for a subset of vertices to be a solid $h$-cover since the following theorem can be proved more easily with a direct argument that avoids explicit reference to solid $h$-covers. Intuitively, a subset of vertices is a solid $h$-cover if it covers all paths of length $h$ and, for each vertex $u$ not in the cover, there is a broadcast protocol for $u$ with enough idle vertices to inform all vertices on a path through $u$ of length at most $h-1$.

Theorem 4. There are broadcast graphs with $2^{k}-2^{j}$ vertices and $\left[\left(2^{k}-2^{j}\right) / 4\right](2 k-j-1)$ edges for which the difference between the maximum and minimum degrees is l for any integers $j, k$, and $l$ with $1 \leq j \leq k-2, k \geq 4$,

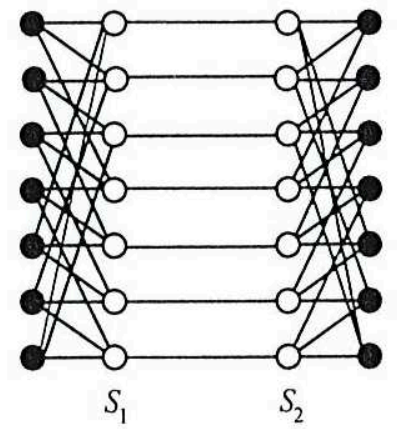

Fig. 6. Solid 2-cover of broadcast graph on $2^{k-1}-4$ vertices. and $1 \leq l \leq j-1$. If $j$ is odd, there are also regular broadcast graphs (i.e., $l=0$ ).

Proof. Let $G$ be a regular bipartite $\mathrm{mbg}$ on $n=2^{k-j+1}$

2 vertices. Choose one of the components of the bipartition to be $S$ and denote the other component $\bar{S}$. $S$ and $\bar{S}$ are both solid 1 -covers for $G$ since both are vertex covers and the degree of $G$ is $\lceil\log n\rceil-1$. Choose nonnegative integers $p$ and $q$ such that $p+q=j-1$ and $p$ $-q=l$.

Construct the graph $G^{\prime}=G_{S}[H]$, where $H$ is the $p$ dimensional hypercube. $G^{\prime}$ is a broadcast graph by Theorem 2. Now construct the graph $G^{\prime \prime}=G_{S}^{\prime}\left[H^{\prime}\right]$, where $H^{\prime}$ is the $q$-dimensional hypercube. Notice that the same graph $G^{\prime \prime}$ is obtained by performing the two compoundings in the other order. In particular, construct $\overline{G^{\prime}}=G_{\bar{S}}\left[H^{\prime}\right]$ and then $G^{\prime \prime}=\bar{G}_{S}^{\prime}[H] . \overline{G^{\prime}}$ is also a broadcast graph by Theorem 2 because $\bar{S}$ is a solid 1-cover for $G$.

Each vertex of $G^{\prime \prime}$ that is in a copy of $S$ has $k-j$ neighbors in its copy of $G$ and $p$ neighbors from the compounding with $H$. Each vertex of $G^{\prime \prime}$ that is in a copy of $\bar{S}$ has $k-j$ neighbors in its copy of $G$ and $q$ neighbors from the compounding with $H^{\prime}$. It is easy to verify that $G^{\prime \prime}$ is a graph with $2^{k}-2^{j}$ vertices, $\left[\left(2^{k}-2^{j}\right) / 4\right](2 k-j$ -1 ) edges, and difference $p-q$ between the maximum and minimum degrees. To complete the proof, we need to show that broadcasting in $G^{\prime \prime}$ can be completed in $k$ $=\lceil\log n\rceil+p+q$ time steps from any originator.

If the originator $u$ is in a copy of $\bar{S}$, it is interconnected with $2^{q}-1$ copies of itself by a $q$-dimensional hypercube $H_{u}^{\prime}$. $u$ first broadcasts in $H_{u}^{\prime}$ so that each copy of $G^{\prime}$ has an informed vertex after $q$ time steps. Since $G^{\prime}$ is a broadcast graph, the broadcast in $G^{\prime \prime}$ can be completed in $\Gamma$ log $n\rceil+p$ more time steps. If the originator $u$ is in a copy of $S$, an analogous argument can be used with $u$ broadcasting first to the $2^{p}$ copies of $\overline{G^{\prime}}$.

At first, it may not be easy to see the solid $h$-cover in the construction above. In fact, the set $\bar{S}^{*}$, which is the union of all copies of $\bar{S}$, is a solid $2^{p}$-cover for $G^{\prime \prime}$. To see this, consider a vertex $u$ which is in $S^{*}$. the complement of $\bar{S}^{*} . u$ is interconnected with $2^{p}-1$ copies of itself by a $p$-dimensional hypercube $H_{u}$ and all of these copies of $u$ are in $S^{*}$. The Hamilton paths of $H_{u}$ are paths of length $2^{p}-1$ containing no vertices of $\bar{S}^{*}$, so $\bar{S}^{*}$ is not a $\left(2^{p}\right.$ $-1)$-cover. Since all other neighbors of $u$ are in $\bar{S}^{*}, \bar{S}^{*}$ is a cover for all paths of length $2^{p}$. If we were to define solid $h$-covers formally and generalize the construction of Theorem 3 to obtain a broadcast protocol based on solid $h$-covers, then the protocol would satisfy a condition similar to Condition 1 of Definition 4.

The construction of Theorem 4 with $l=j-1$ gives the graphs of Khachatrian and Harutounian [10] (see Corollary 1). The graphs of Mahéo and Saclé [13] with 
$2^{k}-8$ vertices result when $j=3$ and $l=0$ (see Corollary 4). All other families of broadcast graphs resulting from Theorem 4 are new families.

\section{4. $k$-WAY SPLITS}

Some previous methods for constructing sparse broadcast graphs are not special cases of the solid cover methods that we have discussed so far. The simplest of these is a method of Farley [6] which constructs a broadcast graph $G$ on $n$ vertices from two broadcast graphs $G_{1}$ and $G_{2}$ with $n_{1}$ and $n_{2}$ vertices such that $n_{1}+n_{2}=n$ and $\left\lceil\log n_{1}\right\rceil=\left\lceil\log n_{2}\right\rceil=\lceil\log n\rceil-1$. Assuming that $n_{1}$ $\geq n_{2}$, a matching with $n_{2}$ edges is added between the vertices of $G_{1}$ and $G_{2}$ to obtain a broadcast graph $G$ with $n$ vertices. Broadcasting in $G$ involves two cases: If the originator $u$ is an endpoint of one of the edges of the matching, it broadcasts first on this edge to a vertex $u^{\prime} . u$ and $u^{\prime}$ now initiate broadcasts in $G_{1}$ and $G_{2}$ which complete in $\lceil\log n\rceil-1$ more time units. If $u$ is not an endpoint of a matching edge, it must be in $G_{1} . u$ initiates a broadcast in $G_{1}$ which completes in $\lceil\log n\rceil-1$ time units. In the last time unit, the edges of the matching are used to inform all vertices of $G_{2}$. This construction proves the following result:

Theorem 5 (Farley). If $n_{1}$ and $n_{2}$ are two integers such that $\left\lceil\log n_{1}\right\rceil=\left\lceil\log n_{2}\right\rceil=\left\lceil\log \left(n_{1}+n_{2}\right)\right\rceil-1$, then $B\left(n_{1}\right.$ $\left.+n_{2}\right) \leq B\left(n_{1}\right)+B\left(n_{2}\right)+\min \left(n_{1}, n_{2}\right)$.

The graphs produced by Farley's construction are not compounds when $G_{1}$ and $G_{2}$ are different, so it is not a special case of our constructions based on solid $h$-covers. (If $G_{1}$ and $G_{2}$ are the same, then the result is a compound relative to the entire set of vertices.) Chau and Liestman [3] gave three constructions which they called 5-way, 6way, and 7-way splits. These constructions are similar Farley's construction and it is possible to include all three split constructions in a more general approach which we call $k$-way split. Our goal is to construct broadcast graphs on $k n+j$ vertices, where $n$ is not a power of 2 and $k$ and $j$ are two integers such that $0 \leq j<k$. We need two definitions before introducing the general $k$-way split construction.

Definition 5 [3]. A graph $G$ on $n$ rertices has an even adjacency split if there exists a partition of the vertices into two dominating sets with cardinalities $\lceil n / 2\rceil$ and $\lfloor n / 2\rfloor$.

Chau and Liestman showed that for each $n$ in the range $2 \leq n \leq 17$ there is a minimum broadcast graph having an even adjacency split. They also showed that all graphs produced by Farley's construction and by their split constructions have even adjacency splits. Since Farley's con- struction can be used to produce a broadcast graph for any $n$, there is at least one broadcast graph with an even adjacency split for each $n$.

Definition 6. A broadcast graph $H=(V(H), E(H))$ on $2 k$ vertices has a broadcast-compatible perfect matching if $H$ has a perfect matching, and for each $x \in V(H)$, there exists a broadcast protocol from vertex $x$ in $H$ such that at least one endpoint of each edge of the matching is aware of the message after $\lceil\log k\rceil$ time steps.

Figure 7 shows two examples of broadcast graphs with broadcast-compatible perfect matchings. The graph and matching in Figure 7(a) are the basis for Chau and Liestman's 5-way split.

Now, let $G_{1}$ and $G_{2}$ be two broadcast graphs on $n$ and $n+1$ vertices, respectively, each having an even adjacency split. We use $S_{1}$ and $\overline{S_{1}}$ to denote the subgraphs induced by the two dominating sets in $G_{1}$. Similarly, $S_{2}$ and $\overline{S_{2}}$ are the subgraphs induced by the dominating sets in $G_{2}$. Let $H=(V(H), E(H))$ be a broadcast graph on $2 k$ vertices having a broadcast-compatible perfect matching.

We construct a graph $G^{\prime}$ using an operation similar to compounding. The difference is that compounding replaces each vertex of $H$ with the same graph, whereas our $k$-way split construction will replace the vertices of $H$ with copies of $S_{1}, \overline{S_{1}}, S_{2}$, and $\overline{S_{2}}$. Viewed another way, our $k$ way split construction replaces each edge of the broadcastcompatible perfect matching in $H$ with a copy of $G_{1}$ or $G_{2}$. More precisely, choose $k-j$ edges of the broadcastcompatible perfect matching in $H$. Replace one endpoint of each of these edges by a copy of $S_{1}$ and the other endpoint by $\overline{S_{1}}$, and join the copies of $S_{1}$ and $\overline{S_{1}}$ with edges in the same way that they are joined in $G_{1}$. Perform a similar operation on the remaining $j$ edges of the perfect matching using $S_{2}$ and $\overline{S_{2}}$. The edges of $H$ which do not belong to the perfect matching are replaced by connections between the subgraphs that have replaced their endpoints. If the two subgraphs have the same number of vertices, they are joined by a perfect matching. If the subgraphs have different numbers of vertices, then the cardinalities of the vertex sets differ by $1 .\lfloor n / 2\rfloor$ vertices of each subgraph

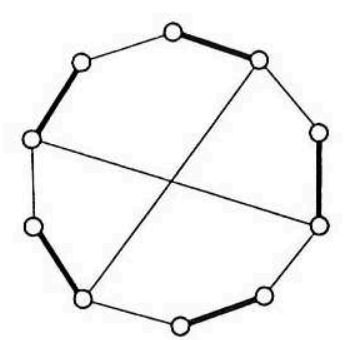

(a)

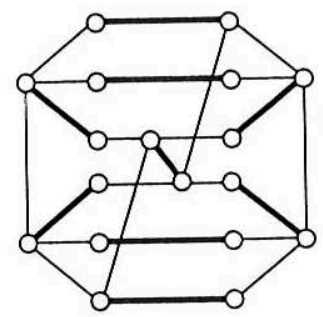

(b)
Fig. 7. Broadcast-compatible perfect matchings. 
are joined by a perfect matching, and the last vertex of the larger subgraph is connected to any vertex of the smaller subgraph. This gives a graph $G^{\prime}$ on $k n+j$ vertices with at most $(k-j)\left|E\left(G_{1}\right)\right|+j\left|E\left(G_{2}\right)\right|+\lceil(n+1) /$ $27(|E(H)|-k)$ edges.

Lemma 1. If $\lceil\log (k n+j)\rceil=\lceil\log k\rceil+\lceil\log n\rceil$, then $G^{\prime}$ is a broadcast graph.

Proof. It is easy to see that $b\left(G^{\prime}\right)=\lceil\log k\rceil+\lceil\log n\rceil$. The originator broadcasts first "in $H^{\prime}$ " using a protocol which ensures that at least one endpoint of each edge of the matching is aware of the message after $\lceil\log k\rceil$ time steps. This ensures that one vertex of each copy of $G_{1}$ or $G_{2}$ is aware of the message after $\lceil\log k\rceil$ time steps. Each of these vertices then broadcasts internally in its copy of $G_{1}$ or $G_{2}$ in $\lceil\log n\rceil$ steps.

The construction above includes the 5-way, 6-way, and 7-way split constructions of Chau and Liestman [3] as special cases which use graphs $H$ with broadcast-compatible perfect matchings on 10,12 , and 14 vertices, respectively. Figure 7(a) shows the graph used for the 5-way split in [3]. Moreover, our construction can be used to construct $k$-way splits for many other values of $k$. For example, Figure 7(b) shows a broadcast graph on 18 vertices with a broadcast-compatible perfect matching which can be used to construct a 9-way split. Notice that the graph in Figure 7(b) is not an mbg.

Farley [6] proposed a second construction which is similar to the $k$-way split construction for $k=3$. This " 3 way split" is not a direct application of Lemma 1 because it uses $H=C_{6}$ which does not have a broadcast-compatible perfect matching. Also, Farley's construction is less restrictive about the sizes of the three graphs than is our construction above. Our construction requires only minor modifications to the broadcast protocol of $G^{\prime}$ and to the way in which spare vertices are interconnected when $n$ is odd to obtain a 3-way split. However, the 6-way split can be used whenever Farley's 3-way split can be used and gives bounds that are at least as good [3]. The $k$-way split construction establishes the following behavior of $B(n)$ :

Theorem 6. Let $n, k$, and $j$ be integers such that $n$ is not a power of $2,0 \leq j<k$, and $\lceil\log (k n+j)\rceil=\lceil\log k\rceil$ $+\lceil\log n\rceil$. If there exist mbg's on $n$ and $n+1$ vertices, each having an even adjacency split, and there exists a minimum broadcast graph on $2 k$ vertices having a broadcast-compatible perfect matching, then

$$
B(k n+j) \leq\left\{\begin{array}{r}
(k-j) B(n)+j B(n+1) \\
\quad+\left\lceil\frac{n+1}{\frac{2}{2}}(B(2 k)-k) \text { if } j>0 ;\right. \\
k B(n)+\left[\frac{n}{2}\right](B(2 k)-k) \quad \text { if } j=0 .
\end{array}\right.
$$

Let us compare the formulas from Corollary 3 and Theorem 6 (with $j=0$ ). We recall that $\mu(n)$ is the minimum size of a solid 2-cover of an mbg on $n$ vertices. If both formulas apply, then the $k$-way split will give a better upper bound if

$$
\mu(n)>\left\lceil\frac{n}{2}\right\rceil \frac{B(2 k)-k}{B(k)} .
$$

For small values of $n$ and $k$, we can check that this formula never holds. For large values, the constructions in [8] and [18] always seem to give better bounds. Moreover, even for $j \neq 0$, using the solid 2-cover construction and applying vertex deletion also seems to give better upper bounds than does the $k$-way split.

The fact that the solid 2-cover method always seems to perform better than does the $k$-way split method is probably a consequence of the smaller matchings used by the solid 2-cover construction. Unfortunately, we have not been able to prove that the solid $h$-cover methods are always better.

\section{COMPARISONS WITH PREVIOUS METHODS}

The values of $B(n)$ for $1 \leq n \leq 15$, and some mbg's realizing these values, were found by Farley et al. [7]. They also showed that hypercubes are mbg's. thus establishing that $B\left(2^{k}\right)=k \cdot 2^{k-1}$. Since then, a few more values of $B(n)$, for small $n$ (and mbg's realizing these values), have been determined [2. 12-14, 19] and several papers, including $[4,14,18,19]$, contain new mbg's for previously known values of $B(n)$. Recently, a second infinite family of mbg's was independently discovered by Khachatrian and Harutounian [10] and by Dinneen et al. [5]. It is interesting that this infinite family, which establishes that $B\left(2^{k}-2\right)=\left(2^{k-1}\right) \cdot(k-1)$, is implicit in an early paper by Knödel [11]. (See [1] for a proof by B. Monien.) The construction methods for this infinite family do not seem to be closely related to our methods. However, notice that a hypercube is a compound of one smaller hypercube into another relative to a solid 0 -cover, i.e., the entire vertex set, of one of the smaller hypercubes.

In 1988, Bermond et al. [2] used four new construction methods to improve most of the bounds for $n \leq 64$ that were known at that time. Most applications of their interconnection of cycles method can be seen as applications of our solid cover methods. A second method, vertex addition, used a restricted version of the idle vertices conditions that we use in this paper and is one of the few previous construction methods to explicitly use information about the broadcast protocols. The vertex deletion method, due to Wang (see [2] or [19]). was used in [2] 
and is also used below to derive new bounds for values of $n$ which are prime or which have "awkward" prime decompositions. The fourth method, cycles with chords, was used in [2] to produce Cayley graphs similar to the family of mbg's with $2^{k}-2$ vertices.

The constructions of Gargano and Vaccaro [8] are based on hypercubes. Their first construction is a vertex deletion method. Their second construction is an application of Theorem 2, with $G$ the mbg on 3 vertices with its solid 1-cover of size 2, and $H$ a hypercube, plus vertex deletion. Their third construction is also an application of Theorem 2 followed by vertex deletion, where $G$ is an mbg on 10 vertices (with a solid 1 -cover of size 8 !) and $H$ is a hypercube. The first construction of Chen [4] is conceptually similar to the second construction in [8]. The difference is that the copies of one vertex of the solid 1cover of the mbg on 3 vertices are interconnected with a graph $H_{1}$ while the copies of the other vertex of the solid cover can use a different graph $\mathrm{H}_{2}$. Since the smaller of $H_{1}$ and $H_{2}$ cannot necessarily be obtained by vertex deletion from the larger, the construction is not a direct application of Theorem 2 and vertex deletion. Chen's subsequent constructions are recursive applications of the first and most of the graphs used are hypercubes.

The constructions of Ventura and Weng in [17] are applications of Theorems 2 and 3 followed by vertex deletion. They divide the values of $n$ between two consecutive powers of 2 into 8 ranges and give a construction with a different graph $G$ for each range. As in [8], $H$ is always a hypercube. For example, in the first range, $G$ is the mbg in Figure 4(b) with 9 vertices and a solid 2-cover of size 3 ; in the second range, $G$ is an mbg with 10 vertices from [14] with solid 2-cover of size 4; and in three other ranges. the graphs in Figures 8-10 are used. In the last range. Ventura and Weng improved the bounds by combining compounding and splitting constructions followed by vertex deletion.

In Section 2, we showed that the main construction method in [10] is a restricted version of our solid 1cover method. Khachatrian and Harutounian also used vertex deletion in [10] for values of $n$ that are not of the form $2^{k}-2^{j}$. In Section 3, we showed that the constructions of [13] involve one application of our solid 1 -cover method and one application of our solid 2-cover method. Theorem 4, which uses solid h-covers. together with vertex deletion, generalizes all of the construction methods in [8, 10, 13, 17], the method in [19] which is vertex deletion from hypercubes, and most of the constructions in [2] and [4]. Our k-way split method generalizes the constructions in [3] and includes restricted versions of Farley's two methods [6] which are variants of 1-way and 3-way splits.

The constructions of Grigni and Peleg [9] are based on hypercubes and generalized Fibonacci numbers. Their constructions produce broadcast graphs with $\mathrm{O}(B(n))$ edges, but, in practice, other methods always seem to use fewer edges. We can see no obvious relationships between our constructions and the constructions in [9].

\section{CONCLUSIONS}

We have presented new methods for constructing sparse broadcast graphs based on graph compounding operations which are relative to vertex sets with certain properties and we have shown that many previous construction methods are applications of our methods. We believe that our methods based on solid covers are always better than is our $k$-way split method, but we have not been able to prove that this is true. An important advantage of our construction methods is that the resulting broadcast protocols are easily verified.

Table I summarizes our results for $1 \leq n \leq 63$. The "Old" values are the best upper bounds on $B(n)$ known to the authors before applying the methods of this paper. The "New" values are obtained using the methods of this paper. Mbg's [and, hence, optimal values of $B(n)$ ] are known for 25 values of $n$ in the range $1 \leq n \leq 63$. Optimal values of $B(n)$ are indicated by *'s in the "Old" column. In this paper, we have improved the bounds on $B(n)$ for nearly half of the 38 values of $n$ for which improvement is possible. Twelve new values are obtained using our constructions based on solid covers, and five more are obtained by applying the vertex deletion method to our new graphs. These new values of $B(n)$ appear in the "New" column of the table and the "Ref." column indicates which method was used. In 15 more cases, indicated by +'s in the "New" column, our solid cover methods give graphs that match the best previous values of $B(n)$. The main advantage of using our methods in these cases is that the broadcast protocols are easy to verify. Comments and construction details related to this table are given in the Appendix.

In addition to the results summarized in Table I, we have applied our methods to several larger values of $n$. Our solid cover constructions improve the upper bounds on $B(n)$ for all $n$ in the range $65 \leq n \leq 105$ except $n=96$ where we matched the bound of [10]. We also compared our methods with those of Farley [6]. Chau and Liestman [3], Gargano and Vaccaro [8], Chen [4]. Grigni and Peleg [9], Ventura and Weng [17], and Khachatrian and Harutounian [10] for $n=513, n=896, n=1008$, and $n=$ 16128. The results are summarized in Table II. When choosing the values of $n$ for Table II, we tried to select values for which most methods apply and for which previous methods exhibit their best behaviors. The values for the methods of Farley and Chau and Liestman that appear in Table II are from [8] and were computed by using the mbg's that were known at that time (i.e., for $n$ $\leq 17$ ) and applying split constructions recursively. The 
values in these two rows can be improved by building on graphs produced by the other methods instead of using recursive splits and small mbg's. These improved values could then be used to improve the results in the "This paper" row, and so on. We have chosen not to perform this recursive computation because it quickly becomes impossible to identify a construction as the result of a particular method. The results in the "Grigni-Peleg" row use a construction from [15] which gives slightly better values than the constructions in [9].

The graphs in the row labeled "This paper" were constructed as follows: The graph with $n=513$ vertices uses
Theorem 3 where $G$ is the 9 vertex graph in Figure 4(b) with a solid 2-cover of size 3 , and $H$ is the graph on 57 vertices from [2]. For $n=896$, Corollary 1 or Theorem 4 with $k=10$ and $j=7$ can be used. The graph with 1008 vertices uses Theorem 2 where $G$ is the 63 vertex mbg from [12] with a solid 1-cover of size 36 , and $H$ is the 4dimensional hypercube. The 16,128 vertex graph is obtained with Theorem 4 where $k=14$ and $j=8$ or with Corollary 1.

Finally, we mention that the question of whether the function $B(n)$ is monotonically increasing between powers of 2 is still an open problem.

TABLE I. Upper bounds on $B(n)$

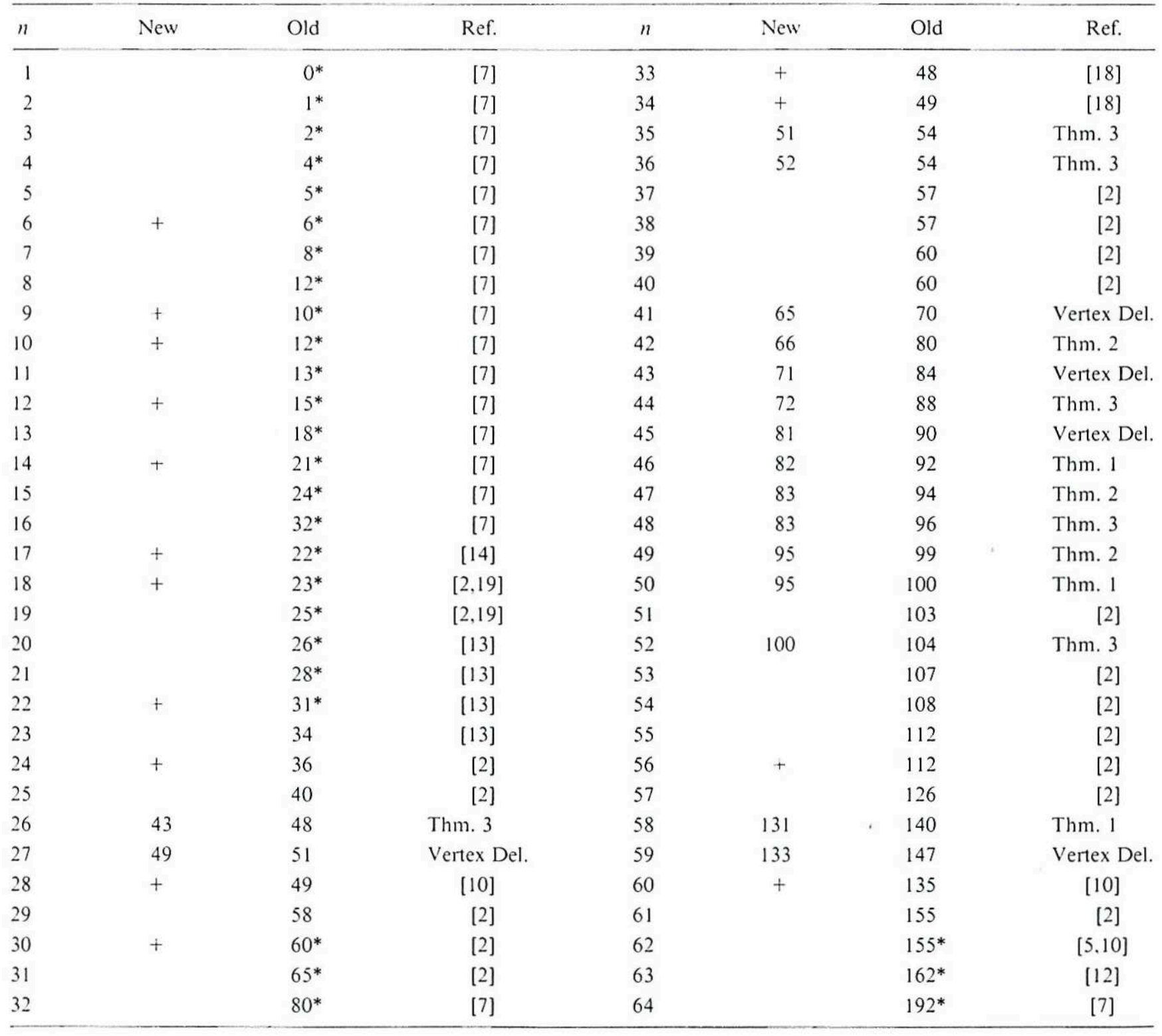


TABLE II. Comparison of upper bounds on $B(n)$ from several methods

\begin{tabular}{lcccr}
\hline Method & $n=513$ & $n=896$ & $n=1008$ & $n=16,128$ \\
\hline This paper & 948 & 2688 & 3744 & 76,608 \\
Khachatrian-Harutounian [10] & 1505 & 2688 & 3780 & 76,608 \\
Weng-Ventura [17] & 1026 & 2880 & 4832 & 108,032 \\
Gargano-Vaccaro [8] & 1408 & 3456 & 4960 & 100,864 \\
Chen [4] & 1368 & 3968 & 4960 & 111,104 \\
Chau-Liestman [3] & 1986 & 4032 & & 112,128 \\
Farley [6] & 1946 & 4288 & 4992 & 112,896 \\
Grigni-Peleg [9] & 1539 & 3584 & 7056 & \\
\hline
\end{tabular}

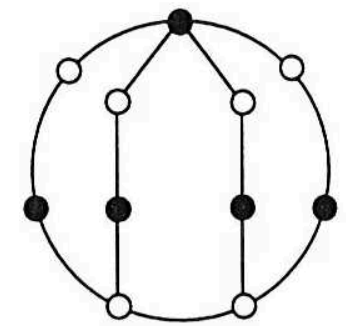

Fig. 8. A solid 2-cover of a broadcast graph on 11 vertices.

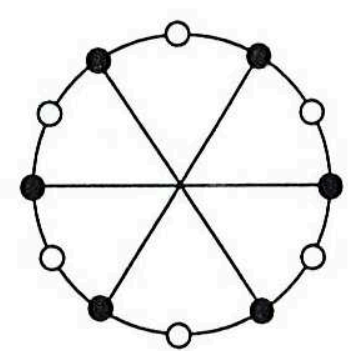

Fig. 9. A solid 1-cover of a broadcast graph on 12 vertices.

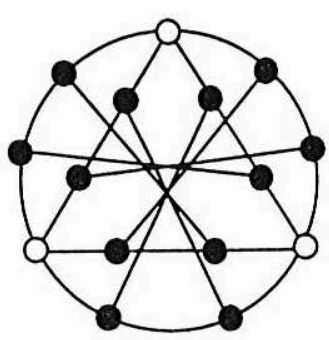

Fig. 10. A solid 1-cover of a broadcast graph on 15 vertices.

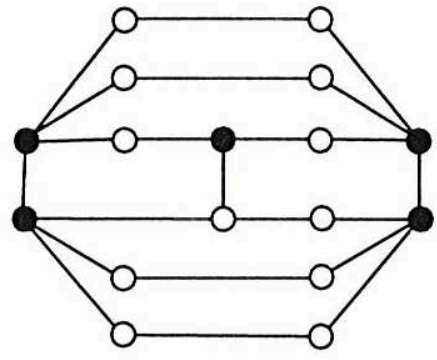

Fig. 11. A solid 2-cover of a broadcast graph on 17 vertices.

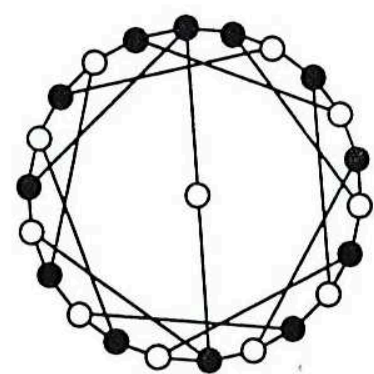

Fig. 12. A solid 1-cover of a broadcast graph on 23 vertices.

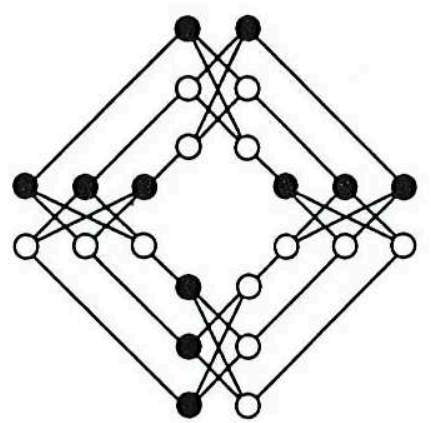

Fig. 13. A solid 2-cover of a broadcast graph on 24 vertices. 


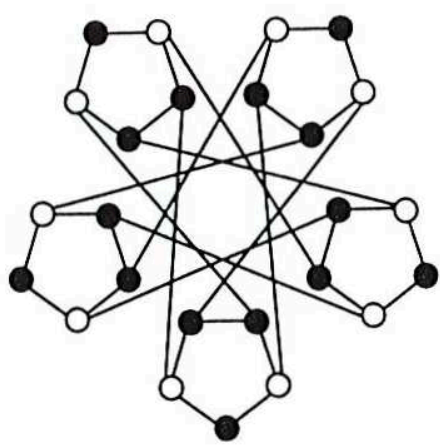

Fig. 14. A solid 1-cover of a broadcast graph on 25 vertices.

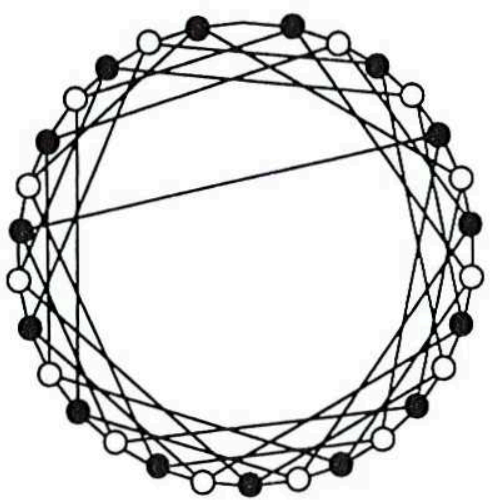

Fig. 15. A solid 1-cover of a broadcast graph on 29 vertices.

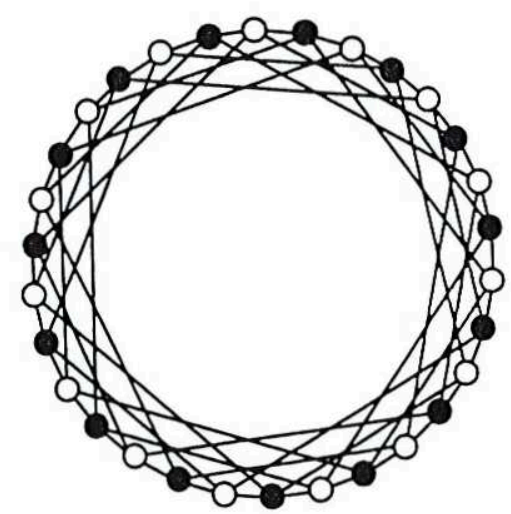

Fig. 16. A solid 1-cover of a broadcast graph on 30 vertices.

We would like to thank Luisa Gargano and Ugo Vaccaro for bringing the results of Khachatrian and Harutounian to our attention and the weather in Vancouver for eliminating distractions that might have delayed the completion of this research.

\section{APPENDIX}

Notation: $K_{n}$ is the complete graph on $n$ vertices, $C_{n}$ is the cycle on $n$ vertices. and $Q_{d}$ is the $d$-dimensional hypercube on $n=2^{d}$ vertices. The vertex deletion method is described in [2] and [19].

- $n=6$ : Theorem 1, where $G$ is the mbg on 3 vertices, gives the $\mathrm{mbg}\left(C_{6}\right)$ in [7].

- $n=$ 9: Theorem 2, where $G$ and $H$ are both the mbg on 3 vertices, gives an $\mathrm{mbg}$ in [6].

- $n=10$ : Theorem 3, where $G=C_{5}$ with a solid 2-cover of size 2 and $H=K_{2}$, gives an mbg in [14].

- $n=12$ : Corollary 1, where $G=C_{6}$ gives an mbg in [10].

- $n=14$ : Theorem 1, where $G$ is the mbg in Figure 3(a) gives an mbg in [18].

- $n=17$ : Vertex deletion from any mbg on 18 vertices.

- $n=18$ : Theorem 3, where $G$ is the graph on 9 vertices in Figure 4(b) with a solid 2-cover of size 3 and $\mathrm{H}=\mathrm{K}_{2}$, gives an mbg in [4].

- $n=22$ : Theorem 3, where $G$ is the mbg on 11 vertices in Figure 8 with a solid 2-cover of size 5 and $\mathrm{H}=\mathrm{K}_{2}$, gives a new mbg.

- $n=24$ : Theorem 1, where $G$ is the mbg on 12 vertices in Figure 9, gives a new broadcast graph. Corollary 1 , where $G=C_{6}$ and $H=Q_{2}$, gives a result in [10]. Corollary 4 gives a 3-regular graph from [2] shown in Figure 13.

- $n=26$ : Theorem 3, where $G$ is the mbg on 13 vertices in Figure 5 with a solid 2-cover of size 7 and $H=K_{2}$. (Note that $G$ is a new mbg on 13 vertices.)

- $n=27$ : Vertex deletion from $n=28$.

- $n=28$ : Corollary 1, where $G$ is the mbg on 14 vertices in Figure 2 and $H=K_{2}$, gives a result in [10].

- $n=30$ : Theorem 1, where $G$ is the mbg on 15 vertices in Figure 10, gives a new mbg.

- $n=33$ : Vertex deletion from the graph below with 34 vertices gives a graph in [18].

- $n=34$ : Theorem 3, where $G$ is the mbg on 17 vertices in Figure 11 with a solid 2-cover of size 5 and $H=K_{2}$, gives a graph in [18]. Note that the mbg of Figure 11 is obtained by vertex deletion from an mbg on 18 vertices constructed with Theorem 3. (See description for $n=18$ above.)

- $n=35$ : Theorem 3. where $G=C_{5}$ with a solid 2-cover of size 2 and $H$ is the mbg on 7 vertices in Figure 3(a). Also by vertex deletion from $n=36$.

- $n=36$ : Theorem 3, where $G$ is the mbg on 9 vertices in Figure 4(b) with a solid 2-cover of size 3 and $H=Q_{2}$.

- $n=41$ : Vertex deletion from $n=42$.

- $n=42$ : Theorem 2, where $G=C_{6}$ and $H$ is an mbg on 7 vertices.

- $n=43$ : Vertex deletion from $n=44$.

- $n=44$ : Theorem 3, where $G$ is the mbg on 11 vertices in Figure 8 with a solid 2-cover of size 5 and $H=Q_{2}$. 
- $n=45$ : Two applications of vertex deletion from $n$ $=47$.

- $n=46$ : Theorem 1, where $G$ is the broadcast graph from [2] on 23 vertices with 35 edges. (See Fig. 12.) Also by vertex deletion from $n=47$.

- $n=47$ : Vertex deletion from the graph on 48 vertices with 84 edges constructed by Theorem 2, where $G=C_{6}$ and $H=Q_{3}$.

- $n=48$ : Theorem 3. where $G$ is the graph on 24 vertices in Figure 13 with a solid 2-cover of size 11 and $\mathrm{H}=\mathrm{K}_{2}$.

- $n=49$ : Theorem 2, where $G$ is the graph on 7 vertices in Figure 3(b) with a solid 1 -cover of size 4 and $H$ is the mbg on 7 vertices in Figure 3(a). Also by vertex deletion from $n=50$.

- $n=50$ : Theorem 1, where $G$ is the graph on 25 vertices in Figure 14 with a solid 1-cover of size 15.

- $n=52$ : Theorem 3, where $G$ is the mbg on 13 vertices in Figure 5 with a solid 2-cover of size 7 and $H=Q_{2}$.

- $n=56$ : Corollary 1, where $G$ is the mbg on 14 vertices in Figure 2 and $H=Q_{2}$, gives a result in [10]. Corollary 4 gives a 4-regular graph from [13].

- $n=58$ : Theorem 1, where $G$ is the graph on 29 vertices in Figure 15 with a solid 1-cover of size 15.

- $n=59$ : From $n=60$ using a modified form of vertex deletion similar to the method used in [2] for $n=29$.

- $n=60$ : Corollary 1 gives a result in [10]. Theorem 1 . where $G$ is the graph on 30 vertices in Figure 16 with a solid 1-cover of size 15 gives a new broadcast graph.

\section{REFERENCES}

[1] J.-C. Bermond and P. Fraigniaud. Broadcasting and NPcompleteness. Technical Report TR 92-01. School of Computing Science. Simon Fraser University (1992).

[2] J.-C. Bermond. P. Hell, A. L. Liestman. and J. G. Peters. Sparse broadcast graphs. Discr. Appl. Math. 36 (1992) 97-130. (Preliminary version published as Technical Report CMPT TR 8804, School of Computing Science, Simon Fraser University, with title "New Minimum Broadcast Graphs and Sparse Broadcast Graphs".)

[3] S. C. Chau and A. L. Liestman. Constructing minimal broadcast networks. J. Comb. Info. Sịst. Sci. 10 (1985) 110-122.

[4] X. Chen. An upper bound for the broadcast function $B(n)$. Chin. J. Comput. 13 (1990) 605-611.
[5] M. J. Dinneen, M. R. Fellows, and V. Faber, Algebraic constructions of efficient broadcast networks. Applied Al. gebra. Algebraic Algorithms and Error Correcting Codes 9. Lecture Notes in Computer Science, Springer-Verlag. Berlin 539 (1991) 152-158.

[6] A. M. Farley. Minimal broadcast networks. Networks 9 (1979) 313-332.

[7] A. M. Farley, S. Hedetniemi, S. Mitchell, and A. Proskurowski. Minimum broadcast graphs. Discr. Math. 25 (1979) 189-193.

[8] L. Gargano and U. Vaccaro, On the construction of minimal broadcast networks. Networks 19 (1989) 673-689.

[9] M. Grigni and D. Peleg. Tight bounds on minimum broadcast networks. SIA.M J. Discr. Math. 4 (1991) $207-$ 222.

[10] L. H. Khachatrian and O. S. Harutounian, Construction of new classes of minimal broadcast networks. Conference on Coding Theory, Armenia (1990).

[11] W. Knödel. New gossips and telephones. Discr. Math. 13 (1975) 95.

[12] R. Labahn. A minimum broadcast graph on 63 vertices. Discr. Appl. Math. 53 (1994) 247-250.

[13] M. Mahéo and J.-F. Saclé, Some minimum broadcast graphs. Technical Report 685, LRI, Université de ParisSud (1991). (Some of the results of this report have since been published with the same title in Discr. Appl. 1/ath. 53 (1994) 275-285).

[14] S. Mitchell and S. Hedetniemi. A census of minimum broadcast graphs. J. Comb. Info. Syst. Sci. 5 (1980) 141 151.

[15] D. Peleg. Tight bounds on minimum broadcast networks. Manuscript (1987).

[16] P. J. Slater, E. Cockayne, and S. T. Hedetniemi. Information dissemination in trees. SIAM J. Comput. 10 (1981) 692-701.

[17] J. A. Ventura and X. Weng, A new method for constructing minimal broadcast networks. Networks 23 (1993) 481-497.

[18] X. Weng and J. A. Ventura, Constructing optimal broadcast networks. Working Paper 91-132. Dept. of Industrial and Management Systems Engineering. Pennsylvania State University (1991).

[19] J. Xiao and X. Wang, A research on minimum broadcast graphs. Chin. J. Comput. 11 (1988) 99-105. 